\title{
A comparative study on hemodynamic outcome of septic patients scheduled for operation either under GA or SAB in low resource settings!
}

\begin{abstract}
Background: Sepsis is global endemic healthcare problem encountered in hospitalized patients all over the world including Bangladesh. Sepsis used to strike an estimated 30 million people worldwide every year, many of whom needlessly die or suffer permanent health issues. Medical resources are strained by the burden of caring for patients suffering from sepsis. Estimated incidence varies from $9.2 \%$ to $21.4 \%$ in different countries of the world. ICU's of low resource settings in developing countries are mostly open type and used to suffer most due to limited resources.
\end{abstract}

Purpose: To share experiences of managing septic patients at low resource settings to create awareness among clinicians thereby adopting right techniques for right patients.

Methods: A prospective randomized controlled study was carried out in Prime Medical College Hospital, Rangpur among 60 patients scheduled for specially abdominal and lower limb surgical emergency cases under general anesthesia (GA Gp) $(n=30)$ as well as by sub arachnoid block (SAB Gp) $(n=30)$ having ASA grade II, III \& IV during the period of April 2014 to April 2015. Results: There were significant differences in preoperative clinical as well as oxygenation state of the patients. In comparison to GA Gp intraoperative Systolic Blood Pressure (SBP) \& Mean Arterial Pressure (MAP) were more profoundly reduced in $\mathrm{SAB} G \mathrm{p}$ which was really difficult to reverse by vasopressors. The underlying reasons of impaired responsiveness were sepsis induced vasodilatation, endothelial dysfunction and venous pooling effect of sympathetic blockade.

Conclusion: Anesthesiologist used to play crucial role not only optimizing patients preoperatively but also maintaining intra operative vital parameters by judicious use of fluids and vaso pressors which materializes successful outcome.

Keywords: sepsis, endemic, resources, arachnoid, vasopressor, intra operative
Volume 2 Issue 5 - 2015

\section{Lt Colonel Abul Kalam Azad}

Department of anesthesiology and intensive care, Combined Military Hospital, Bangladesh

Correspondence: Lt Colonel Abul Kalam Azad, Classified anesthesiologist, Dept of anesthesiology and intensive care, Combined Military Hospital, Dhaka-1206, Bangladesh, Tel 0088017I5010956, Email azad100864@gmail.com

Received: May 22, 2015 | Published: August 12, 2015
Abbreviations: UTI, urinary tract infections; ICU, intensive care unit; GA, general anesthesia; SAB, sub arachnoid block; MAP, mean arterial pressure; SBP, systolic blood pressure; GP, general pratitioner

\section{Introduction}

Nosocomial infection and sepsis is global endemic healthcare problem encountered in hospitalized patients all over the world including Bangladesh. ${ }^{1}$ In the developing countries, sepsis remains a leading cause of death. Sepsis strikes an estimated 30million people worldwide every year, many of whom needlessly die or suffer permanent health issues. Medical resources are strained by the burden of caring for patients suffering from sepsis. ${ }^{2,3}$

The rate of NI varies from $9.2 \%$ to $21.4 \%$ in different countries of the world. A cross sectional study conducted in 1991 in the surgical wards of Dhaka Medical College Hospital showed that out of 240 patients, $72(38.0 \%)$ suffered from NI of which $36.1 \%$ had wound infection and $23.6 \%$ had UTI. Prevalence of NI in post-operative patients was found to be higher $(49.0 \%)$ than preoperative patients $(15.9 \%) .{ }^{1,4}$ As low resource settings ICU's in developing countries are mostly open type due to limited staffing, lack of protocol and if having protocol at all, then reluctant to stick with protocol and compliance. ${ }^{5}$. Considering type of ICU and environmental pollution patients are vulnerable to infection and sepsis. Low resource setting typically defined as lack of fund to cover healthcare cost. ${ }^{6}$
Awareness, education, resources mobilization and timely intervention are seriously needed to prevent, diagnose and treat sepsis early. So, the purpose of the study is to share experiences of managing septic patients at low resource settings and to create awareness thereby adopting right technique for right patient.

\section{Methods}

This prospective randomized controlled study was carried out in Prime Medical College Hospital, Rangpur among 60 patients. Study was conducted among septic patients specially abdominal and lower limb surgical cases scheduled for emergency surgery under general anesthesia (GA) as well as by sub arachnoid block(SAB) having ASA grade II, III \& IV during the period of April 2014 to April 2015. There were limited scope of avoiding surgery as because proposed surgery was for source control and patients already reported late for that operation became inevitable and urgent in nature; moreover legal guardians counseled and consented to operate the case. Pre-anesthesia checkup was mostly done either in ICU or theatre. Clinical status of the patients was assessed considering variables in (Table 1).

Exclusion criteria were coagulopathy, hypovolemia and hypotension, sepsis at the site of injection. Comparatively less toxic and better hemodynamic state patients were selected for SAB and relatively unprepared and uncontrolled patients were selected for general anesthesia. Pulse Rate, Systolic Blood Pressure (SBP), Mean Arterial Pressure (MAP) and urine output were taken as variables 
to assess hemodynamic state of patients (Tables $2 \& 3$ ). In general anesthesia (GA) groups $(\mathrm{n}=30)$, patients fulfilled selection criteria and relatively unprepared, uncontrolled patients were taken. All patients were administered same general anesthetics less few exceptions. In sub arachnoid block groups $(\mathrm{SAB})(\mathrm{n}=30)$, patients fulfilled selection criteria and relatively better hemodynamic were taken. All patients were administered same local anesthetics less few exceptions.

Table I Variables for assessing Preoperative Clinical State of Patients

Mental State
Temperature
Pulse Rate
SBP
MAP
Respiratory rate
Urine output
Capillary refill
Hypoxia

Table 2 Inclusion criteria for the patients with abdominal or lower limb sepsis were raised temperature and increased WBC counts

\begin{tabular}{ll}
\hline Variables & Values \\
\hline Temperature & $<36{ }^{\circ} \mathrm{C}$ or $>38^{\circ} \mathrm{C}$ \\
WBC Counts & $<4 \times 10^{\circ} / \mathrm{L}$ or $\quad>12 \times 10^{9} / \mathrm{L}$ \\
\hline
\end{tabular}

Table 3 Inclusion criteria for the patients with abdominal or lower limb sepsis were raised pulse rate and increased Urine output

\begin{tabular}{ll}
\hline Variables & Normal Values \\
\hline Pulse Rate & $60-90 / \mathrm{min}$ \\
SBP & $90-140 \mathrm{~mm}$ of $\mathrm{Hg}$ \\
MAP & $70-105 \mathrm{~mm}$ of $\mathrm{Hg}$ \\
Urine Output & $5-1 \mathrm{ml} / \mathrm{kg} / \mathrm{hr}$ \\
\hline
\end{tabular}

Primary clinical assessment, evaluation and laboratory reports were screened before surgery. Along with evaluation, simultaneous resuscitation were carried out, legal guardian was counseled about consequences and probable outcome of the patient. With that patients put on table, basic monitoring equipments were established and measured, patency \& adequacy of IV cannulae assessed. Careful selection of anesthetics was made to get better outcome. Anesthetic managements were formulated considering protocol of Surviving Sepsis Campaign and guidelines of anesthesia for emergency surgery. While administering GA, proper denitrogenation, step wise slow IV induction, ventilation with protective lung strategies thereby avoidance of lung injury, judicious use of fluid, vasopressors and optimizing volume and $\mathrm{SAB}$ was undertaken with caution keeping in mind the hemodynamic instability might be very detrimental. Periodical intraoperative monitoring and measurement of vital parameters were done and episodes of hypotension were managed with whole blood, colloids, and crystalloids and appropriate vasopressors mostly by $\alpha 1$ agonist and other medications whatever drugs or strategies considered best as per scenario. Per operative hemodynamic parameters were recorded in separate forms for $\mathrm{GA}$ and $\mathrm{SAB}$. At the end of the operation, patients were kept in ICU for close monitoring, empathic nursing as well as for appropriate therapy.

\section{Results}

A total 60 patients were included in this study. Evaluating clinical variables and preoperative clinical state, there were significant differences in pulse rate; systolic blood pressure (SBP), mean arterial pressure (MAP), and capillary re-fill as well as state of oxygenation and urine output (Table 4). There were significant differences in preoperative state of oxygenation between two groups (Table 5). In comparison to preoperative value, intraoperative SBP was dropped down $14.08 \%$ in GA Gp which was dropped into $25.89 \%$ in SAB Gp without vasopressors. Though declined value was elevated respectively $15.15 \% \& 14.21 \%$ into targeted value using vasopressors (Tables 6 \& 7).

In comparison to preoperative value, intraoperative MAP was dropped down $27.28 \%$ in GA Gp which was dropped into $43.576 \%$ in SAB Gp without vasopressors. Though declined value was elevated respectively $5.92 \% \& 20.27 \%$ into targeted value using vasopressors (Tables 6 \& 7). In comparison to preoperative value, intraoperative pulse rate reduced to $7.69 \%$ in GA Gp though it was increased $44.68 \%$ in SAB Gp without vasopressor. Although both values were reduced by $8.33 \%$ in GA Gp and $17.64 \%$ in SAB Gp respectively with vasopressor (Tables 8 \& 9).

In comparison to GA Gp, though SBP could be elevated up to optimum level $(\mathrm{SBP}=14.08$ to $15.15 \%$ \& $\mathrm{MAP}=27.28$ to $5.92 \%$ ) using vasopressors but due to impaired responsiveness reversing dropped down value ( $\mathrm{SBP}=25.89$ to $14.21 \& \mathrm{MAP}=43.57$ to $20.27 \%$ ) of MAP in SAB Gp was really difficult (Tables 8 \& 9). In comparison to preoperative value, intraoperative urine output was elevated $53.65 \%$ in GA group whereas it was dropped into $22.64 \%$ in SAB Gp. Though declined value SAB Gp again elevated into $56.86 \%$ and GA Gp further elevated into $109.52 \%$ (Table 8 \& 9). There were no such significant differences in pulse rate, SBP and MAP other than urine output in the early postoperative period (Table 10).

Table 4 Comparison of preoperative clinical state of patients between two groups $(n=60)$

\begin{tabular}{llll}
\hline Variables & Group GA (n=30) & Group SAB (n=30) & p value \\
\hline Mental State & & & - \\
Conscious & $30(100.0 \%)$ & $30(100.0 \%)$ & $0(0.0 \%)$ \\
Unconscious & $0(0.0 \%)$ & $99.6 \pm 1.30$ & 0.12 \\
Temperature $\left({ }^{\circ} \mathrm{C}\right)$ & $100.2 \pm 1.45$ & $88.60 \pm 6.63$ & $<0.001 *$ \\
Pulse Rate $(\mathrm{per} / \mathrm{min})$ & $104.03 \pm 0.96$ & & \\
\hline
\end{tabular}

Statistical analysis was accomplished by Unpaired' test to compare between groups.

$\mathrm{n}=\quad$ Total number of subjects

$* \quad$ Significant

Table 5 Comparison of preoperative state of oxygenation between two groups $(n=6)$

\begin{tabular}{|c|c|c|c|}
\hline SBP $(\mathrm{mmHg})$ & $101.0 \pm 12.28$ & I I $0.5 \pm 7.80$ & $0.001 *$ \\
\hline $\mathrm{DBP}(\mathrm{mmHg})$ & $70.83 \pm 12.32$ & $16.40 \pm 3.80$ & $<0.00 I^{*}$ \\
\hline Respiratory rate (per/min) & $20.37 \pm 2.95$ & $88.33 \pm 10.11$ & $<0.001 *$ \\
\hline
\end{tabular}


Table Continued...

\begin{tabular}{|c|c|c|c|}
\hline SBP (mmHg) & $101.0 \pm 12.28$ & $I 10.5 \pm 7.80$ & $0.001 *$ \\
\hline Oliguria (ml/kg) & $0.30 \pm 0.11$ & $16.40 \pm 3.80$ & $<0.00 I^{*}$ \\
\hline Capillary Re-fill (sec) & & $0.50 \pm 0.13$ & $<0.001^{*}$ \\
\hline \multicolumn{4}{|l|}{ Hypoxia } \\
\hline Mild & $4.10 \pm 1.16$ & $2.01 \pm 1.0$ & $<0.001 *$ \\
\hline
\end{tabular}

No hypoxia

Statistical analysis was accomplished by Chi-square test to compare between groups.

$\mathrm{n}=\quad$ Total number of subjects

$* \quad$ Significant

Table 6 Comparison of intraoperative hemodynamic variables of patients without vasopressor between two groups ( $\mathrm{n}=60$ )

\begin{tabular}{|c|c|c|c|}
\hline Variables & $\begin{array}{l}\text { Group GA }(n=30) \\
\text { Mean } \pm \text { SD }\end{array}$ & $\begin{array}{l}\text { Group SAB }(n=30) \\
\text { Mean } \pm \text { SD }\end{array}$ & p value \\
\hline Pulse Rate (per/min) & $96.4 \pm 3.41$ & $|36.56 \pm| \mid .85$ & $<0.00 I^{*}$ \\
\hline $\mathrm{SBP}(\mathrm{mmHg})$ & $90.2 \pm 7.12$ & $80.21 \pm 6.45$ & $<0.001 *$ \\
\hline MAP $(\mathrm{mmHg})$ & $56.1 \pm 4.36$ & $50.2 \pm 5.34$ & $<0.001 *$ \\
\hline Urine output (ml/kg) & $0.51 \pm 0.12$ & $0.40 \pm 0.11$ & $0.001 *$ \\
\hline
\end{tabular}

Statistical analysis were done by Unpaired ' $\mathrm{t}$ ' test was performed to compare between groups.

$\mathrm{n}=\quad$ Total number of subjects

$* \quad$ Significant

Table 7 Comparison of intraoperative hemodynamic variables of patients with vasopressor between two groups ( $n=60$ )

\begin{tabular}{llll}
\hline Variables & $\begin{array}{l}\text { Group GA }(\mathbf{n}=30) \\
\text { Mean } \pm \text { SD }\end{array}$ & $\begin{array}{l}\text { Group SAB (n=30) } \\
\text { Mean } \pm \text { SD }\end{array}$ & p value \\
\hline Pulse Rate (per/min) & $88.22 \pm 6.54$ & $112.2 \pm 10.29$ & $<0.00 I^{*}$ \\
SBP $(\mathrm{mmHg})$ & $102.42 \pm 9.65$ & $90.54 \pm 8.44$ & $<0.00 I^{*}$ \\
MAP (mmHg) & $65.56 \pm 5.98$ & $60.33 \pm 6.47$ & $0.002^{*}$ \\
Urine output (ml/kg) & $1.01 \pm 0.31$ & $0.51 \pm 0.29$ & $<0.00 I^{*}$ \\
\hline
\end{tabular}

Statistical analysis were done by Unpaired ' $t$ ' test was performed to compare between groups.

$\mathrm{n}=$ Total number of subjects

$*$ Significant

Table 8 Mean \% changes of hemodynamic variables from preoperative to intraoperative value between two groups

\begin{tabular}{llll}
\hline Variables & $\begin{array}{l}\text { Group GA }(\mathbf{n = 3 0}) \\
\text { Mean } \pm \text { SD }\end{array}$ & $\begin{array}{l}\text { Group SAB }(\mathbf{n}=30) \\
\text { Mean } \pm \text { SD }\end{array}$ & p value \\
\hline Pulse Rate $(\mathrm{per} / \mathrm{min})$ & $7.69 \pm I .47$ & $44.68 \pm 4.55$ & $<0.00 I^{*}$ \\
SBP $(\mathrm{mmHg})$ & $14.08 \pm 3.76$ & $25.89 \pm 6.71$ & $<0.00 I^{*}$ \\
MAP $(\mathrm{mmHg})$ & $27.28 \pm 5.13$ & $43.57 \pm 8.79$ & $<0.00 I^{*}$ \\
Urine output $(\mathrm{ml} / \mathrm{kg})$ & $53.65 \pm 8.54$ & $22.64 \pm 4.23$ & $<0.00 I^{*}$
\end{tabular}

Statistical analysis were done by Unpaired ' $t$ ' test was performed to compare between groups.

$\mathrm{n}=\quad$ Total number of subjects

$* \quad$ Significant

Table 9 Mean \% changes of hemodynamic variables from intra-operative dropped value to intra-operative targeted value between two groups ( $\mathrm{n}=60$ )

\begin{tabular}{llll}
\hline Variables & $\begin{array}{l}\text { Group GA (n=30) } \\
\text { Mean } \pm \text { SD }\end{array}$ & $\begin{array}{l}\text { Group SAB (n=30) } \\
\text { Mean } \pm \text { SD }\end{array}$ & p value \\
\hline Pulse Rate (per/min) & $8.33 \pm 1.41$ & $17.64 \pm 2.11$ & $17.64 \pm 2.11$ \\
SBP $(\mathrm{mmHg})$ & $15.15 \pm 2.44$ & $14.21 \pm 2.84$ & $14.21 \pm 2.84$ \\
MAP (mmHg) & $5.92 \pm 1.97$ & $20.27 \pm 4.12$ & $20.27 \pm 4.12$ \\
Urine output $(\mathrm{ml} / \mathrm{kg})$ & $109.52 \pm 6.56$ & $56.86 \pm 4.59$ & $56.86 \pm 4.59$ \\
\hline
\end{tabular}

Statistical analysis were done by Unpaired ' $t$ ' test was performed to compare between groups.

$\mathrm{n}=\quad$ Total number of subjects

$* \quad$ Significant

Table 10 Comparison of hemodynamic variables in postoperative period between two groups $(n=60)$

\begin{tabular}{|c|c|c|c|}
\hline Variables & Group GA(n=30) & Group SAB $(n=30)$ & $P$ value \\
\hline Pulse Rate (per/min) & $82.2 \pm 12.7$ & $82.2 \pm 12.7$ & $0.15 \mathrm{~ns}$ \\
\hline $\mathrm{SBP}(\mathrm{mm}$ of $\mathrm{Hg})$ & || $4.5 \pm 14.2$ & || $4.5 \pm \mid 4.2$ & $0.1 \mathrm{Ins}$ \\
\hline MAP (mm of $\mathrm{Hg})$ & $76.3 \pm 6.5$ & $76.3 \pm 6.5$ & $0.001 *$ \\
\hline Urine output ( $\mathrm{ml} / \mathrm{min})$ & $0.75 \pm .02$ & $0.75 \pm .02$ & $<0.00 I^{*}$ \\
\hline
\end{tabular}

Statistical analysis were done by Unpaired ' $t$ ' test was performed to compare between groups.

$\mathrm{n}=\quad$ Total number of subjects

$* \quad$ Significant

Citation: Azad LCAK.A comparative study on hemodynamic outcome of septic patients scheduled for operation either under GA or SAB in low resource settings! J Anesth Crit Care Open Access. 2015;2(5): I-6. DOI: I0.15406/jaccoa.20 I5.02.00072 


\section{Discussion}

To estimate and judge the hemodynamic outcome of septic patients scheduled for operation either under GA or SAB in low resource settings, this study was conducted at Prime Medical College Hospital, Rangpur among 60 patients. Sepsis is more common than heart attack, and claims more lives than any cancer, yet even in the most developed countries fewer than half of the adult population have heard of it. In the least developed countries, sepsis remains a leading cause of death. Colloquially known as "blood poisoning", sepsis is a life-threatening medical condition that arises when the body's attempt to fight an infection results in the immune system damaging tissues and organs causes widespread inflammation, leaky blood vessels resulting blood pressure drops and abnormal blood coagulation resulting in organ damage, multiple organ failures ensue. ${ }^{3}$ MAP considered being the perfusion pressure seen by organs in the body. ${ }^{7}$ So is the necessity of maintaining MAP and creating awareness among concern physicians.

Limitation of the study was not to invasively measure SBP and MAP, failure to conduct multicentre study with a larger sample size and failure to do blood culture. Considering socioeconomic status and awareness patients used to report late towards hospital so is the state of clinical condition of the patients. Preoperative state of oxygenation is poor in patients belongs to GA Gp as the overall clinical state and peripheral perfusion is insufficient which is compatible with Seok Hwa Yoon ${ }^{8}$ study. Increased pulse rate and significant reduction of SBP and MAP in SAB Gp in comparison to GA Gp due to combined effect of sepsis induced cardiac and vascular dysfunction (vasoplegia), sympathetic block by $\mathrm{SAB}$ and systemic effects of induction agent which is consistent with Huntter JD et al. ${ }^{9}$ Eissa D et al. ${ }^{10}$ Thooft et al. ${ }^{11}$ Young J D. ${ }^{12}$ \& Reich DL et al. ${ }^{13}$ Then reduced pulse rate and dropped down value of SBP and MAP was increased up to optimum perfusion pressure level due to vasoactive effect of vasopressors which is consistent with Thooft et al. ${ }^{11}$ study. More profound cardiovascular instability in SAB Gp is due to inefficient compensatory vasoconstriction by sepsis induced vasodilatation, endothelial dysfunction superimposed with venous pooling by sympathetic blockade which is compatible with D Eissa. ${ }^{10}$, Cosimo Chelazzi. ${ }^{14}$ \& Spencer S Liu et al..$^{15}$

Maintaining optimum MAP and intravascular volume is essential for renal protection and urine output, in SAB Gp reduced urine output again increased along with elevated MAP which is compatible with Elizabeth Cordes-Behringer et al. ${ }^{16}$

\section{Conclusion}

Patients with sepsis scheduled for source control operation either under GA or SAB is always challenging. Role of anesthesiologist is crucial, considering preoperative resuscitation aimed at optimizing vital organ perfusion, careful intraoperative management aimed at maintaining optimum volume status, avoidance of lung injury during ventilation, maintaining blood chemistry and beat to beat monitoring can only lead to successful outcome.

\section{Acknowledgements}

None.

\section{Conflicts of interest}

The authors declare that there are no conflicts of interest.

\section{Funding}

None.

\section{References}

1. Mohiuddin Md, J Ashraful Haq, Mozammel Hoq Md, et al. Microbiology of Nosocomial Infection in Tertiary Hospitals of Dhaka City and Its Impact. Bangladesh Journal of Medical Microbiology. 2010;4(2):32-38.

2. Angus DC, van der Poll T. Severe sepsis and septic shock. N Engl J Med. 2013;369(9):840-851.

3. www.world-sepsis-day.org/?MET=SHOWCONTAINER ...11

4. Mohammad Murshed, Saima Kamar. Organisms in operative site in an urban hospital in Dhaka city: An urgent need to develop an infection control program in Bangladesh. Bangladesh Journal of Medical Microbiology. 2013;7(1):20-24.

5. Manimala Rao S, Suhasini T. Organization of Intensive Care Unit and Predicting Outcome of Critical Illness. Indian $J$ Anaesth. 2003;47(5):328-337.

6. Design for High - and Low - Resource Settings. BIOEN 404 Team Design I. 2014.

7. Mean arterial pressure

8. Yoon SH. Concerns of the anesthesiologist: anesthetic induction in severe sepsis or septic shock patients. Korean J Anesthesiol. 2012;63(1):3-10.

9. Hunter JD, Doddi M. Sepsis and the heart. Br J Anaesth. 2010;104(1):311.

10. Eissa D, Carton EG, Buggy DJ (2010) Anaesthetic management of patients with severe sepsis. Br J Anaesth. 2010;105(6):734-743.

11. Thooft, Favory R, Salgado DR, et al. Effects of changes in arterial pressure on organ perfusion during septic shock. Critical Care. 2011;15 (5):R222.

12. Young JD. The heart and circulation in severe sepsis. Br J Anaesth. 2004;93(1):114-120.

13. Reich DL, Hossain S, Krol M, et al. Predictors of hypotension after induction of general anesthesia. Anesth Analg. 2005;101(3):622-628.

14. Chelazzi C, Villa G, Mancinelli P, De Gaudio AR, Adembri C (2015) Glycocalyx and sepsis-induced alterations in vascular permeability. Crit Care. 2015;19:26.

15. Spencer S Liu. Current issues in spinal anesthesia. Can J Anesth. 2002;49(1):R36-R40.

16. Eizabeth Cordes Behringer. Oliguria: Perioperative management. Anestesiologia. 2006;29(Suppl 1):S21-S26. 